Erratum

\title{
Phosphoglycerate Mutase 1 Predicts the Poor Prognosis of Oral Squamous Cell Carcinoma and is Associated with Cell Migration - Erratum
}

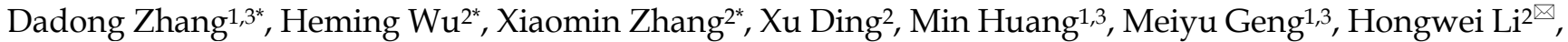
Zuoquan $\mathrm{Xie}^{1,3 \bowtie}$

1. Division of Anti-tumor Pharmacology, State Key Laboratory of Drug Research, Shanghai Institute of Materia Medica, Chinese Academy of Sciences, Shanghai 201203, China.

2. Jiangsu Key Laboratory of Oral Diseases, Nanjing Medical University; Department of oral and maxillofacial surgery, Affiliated Hospital of Stomatology, Nanjing Medical University, Nanjing 210029, China.

3. University of Chinese Academy of Sciences, Beijing 100049, China.

${ }^{*}$ Contributing equally to this article.

$\bowtie$ Corresponding author: Zuoquan Xie, zqxie@simm.ac.cn; Telephone: 86-21-50806600-2413. Hongwei Li, lhwqhxa@njmu.edu.cn.

(C) The author(s). This is an open access article distributed under the terms of the Creative Commons Attribution License (https://creativecommons.org/licenses/by/4.0/). See http://ivyspring.com/terms for full terms and conditions.

Published: 2020.01.24

Corrected article: J Cancer 2017; 8(11): 1943-1951. doi: 10.7150/jca.19278.

In our paper [1], the Figure 2C, Figure 2E and Figure 4B should be corrected as the following Figure A1, Figure A2 and Figure A3.

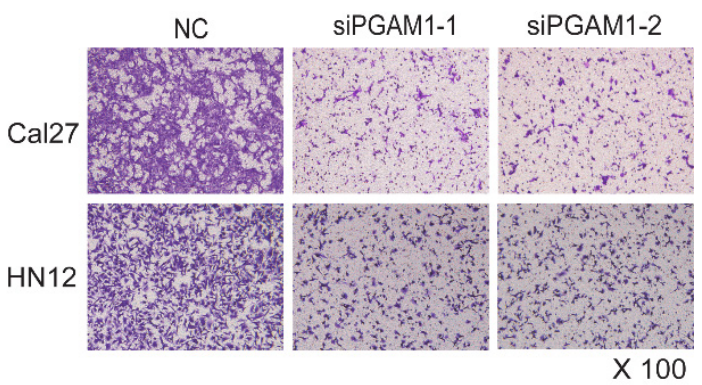

Figure Al. (Figure 2C). Decreased cell migration after knocking down PGAMI. Cell migration (24 h) was dramatically decreased after knocking down PGAM1 in both Cal27 and HN12 cells.
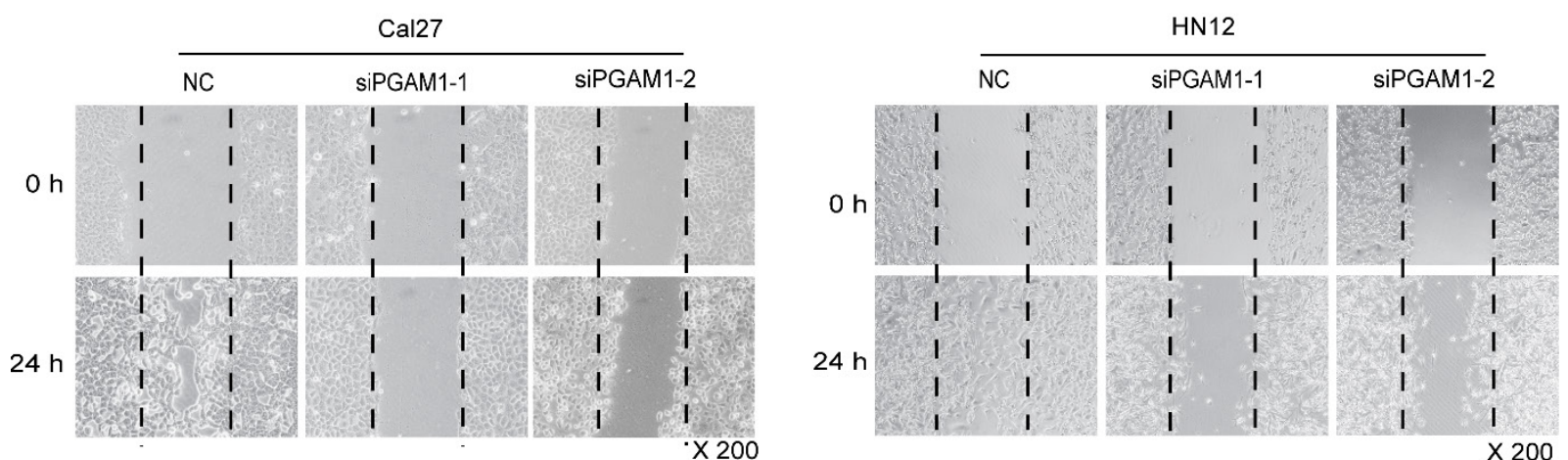

Figure A2. (Figure 2E). Decreased cell mobility after knocking down PGAM1. Cell mobility was decreased after knocking down of PGAMI in both Cal27 and HN12, as determined by the wound-healing assay. 


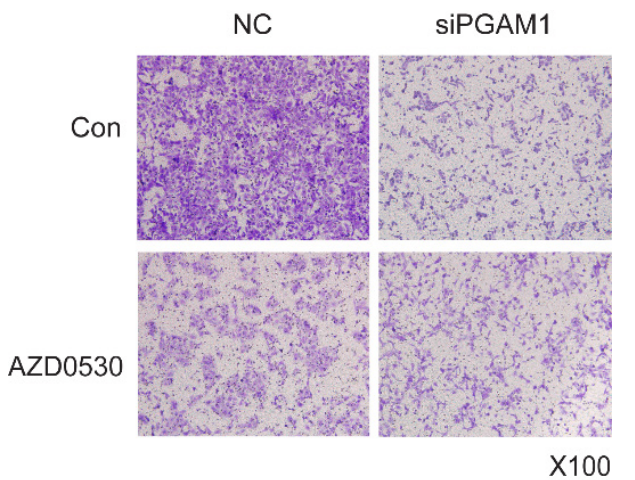

Figure A3. (Figure 4B). SRC phosphorylation is required for PGAM1-mediated cell migration. Cell migration was decreased by AZD0530 or PGAMI knock down, while pretreatment with AZD0530 abolished a further decrease in cell migration when PGAMI was knocked down.

\section{References}

[1] Zhang D, Wu H, Zhang X, Ding X, Huang M, Geng M, Li H, Xie Z. Phosphoglycerate Mutase 1 Predicts the Poor Prognosis of Oral Squamous Cell Carcinoma and is Associated with Cell Migration. J Cancer. 2017;8(11):1943-1951. doi: 10.7150/jca.19278. 